\title{
Approximation Algorithm for Optimal Wiring on Rectangular Structure
}

\author{
Pawan Kumar Patel \\ (Department of Computer \\ Science \& Engineering), \\ IIT Kanpur India
}

\author{
Manoj Diwakar \\ (Department of Computer \\ Science \& Engineering), \\ DIT University Dehradun, \\ India
}

\author{
Kunal Gupta \\ (Department of Information \\ technology), \\ HCT, Muscat, Oman
}

\author{
Amrendra Tripathi \\ (Department of Computer \\ Science \& Engineering), \\ DIT University Dehradun, \\ India
}

\begin{abstract}
In this paper we discuss our attempts to find an approximation for the optimal wiring on rectangular structure. Here we are given a rectangle partitioned into smaller rectangles by axisparallel line segments. Find a subset of the segments such that the resulting structure from these segments is connected and it touches every smaller rectangles. Although these attempts have not yielded any satisfactory result but gives direction to solve this problem need a very different approach.
\end{abstract}

\section{Keywords}

Graph theory, Approximation algorithm, Steiner tree, wiring on rectangular structure.

\section{INTRODUCTION}

Given a rectangle partitioned into smaller rectangles by horizontal and vertical line segments, find a set of the linesegments which touches each rectangle at least at one point on its boundry and these segments are connected (i.e., there is a path between any two points of these segments ). The optimization criterion is to minimize the sum of lengths of these segments.

\section{APPROXIMATION BY REDUCING TO STEINER TREE PROBLEM}

The trivial solution for the problem is to include the entire graph in the solution. The cost of this solution is $(L+W)+$ $\sum_{i}\left(l_{\mathrm{I}+} \mathrm{w}_{\mathrm{i}}\right)$ where $\mathrm{l}_{\mathrm{i}}$ and $\mathrm{w}_{\mathrm{i}}$ are the length and the width of the ith rectangle, and $\mathrm{L}$ and $\mathrm{W}$ are the length and the width of the entire floorplan.

Here we present a simple strategy that gives us a first solltion to the wiring problem.

For convenience treat it as a graph where the vertex set is the collection of the corners of all the rectangles and edges are the line segments between the vertices in the floor plan. A side is a line segment which connects two corners of the same rectangle. In general a side may contain more than one edge. The edges are weighted by their lengths. Enhance this graph to $G$ where add a vertex $u_{i}$ to $G$ for each face $f_{i}$. Connect $u_{i}$ to each vertex on the boundry of $f_{i}$ and weight each of these edges with $0.5\left(l_{i}+w_{i}\right)$, see figure 1 . Now compute an approximation for steiner tree for $\left\{u_{i}\right\}_{i}$ Let its cost be c. Note that each leaf vertex of the steiner tree must be a $u_{i}$ vertex (converse may not be true). Further, modify the tree as follows. If any $u_{i}$ has degree greater than 1 , then replace $u_{i}$ and include the sides of that rectangle to maintain the connectivity. Observe that the cost of the solution will not exceed since two of the new edged cost $\left(l_{i}+w_{i}\right)$. The resulting tree is a subgraph of $\mathrm{G}$ and it must touch all the faces therefore it is a potential solution.

Let $t$ is the optimal cost of the wiring solution. By adding an edge from each $u_{i}$ to one of the vertices in the solution gives a candidate for the steiner tree for $\left\{u_{i}\right\}_{i}$. Robins and Zelikovsky [1] have offered a 1.55-factor approximation for the steiner tree problem. Thus $\mathrm{c} \leq 1.55 \mathrm{t}+0.5 \sum_{i}\left(l_{\mathrm{I}+} \mathrm{W}_{\mathrm{i}}\right)$.

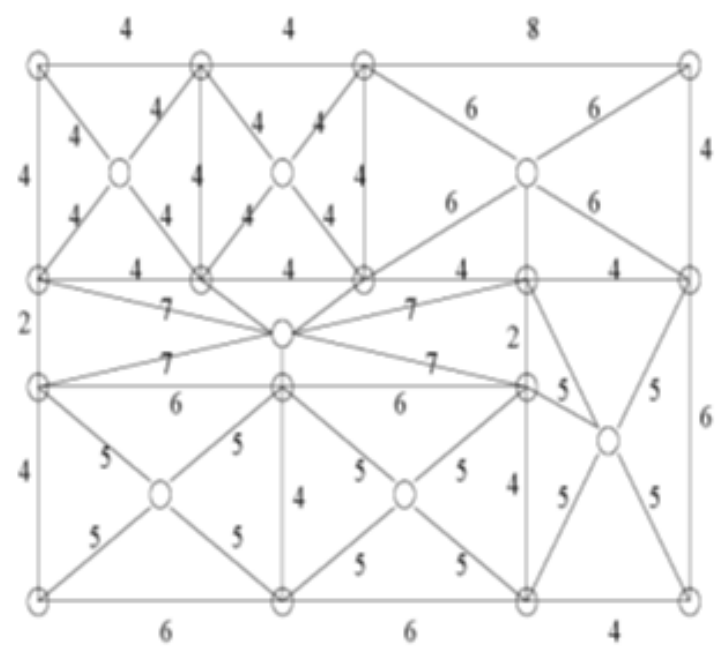

Figure 1: Transformed graph for rectangular wiring problem

\section{PRIMAL-DUAL APPROACH}

Goemans and Williamson [2] discussed generic problem, called constrained forest problem. It is the generalization of several problems such as steiner tree, $\mathrm{T}$-join, perfect matching, exact-partitioning etc. It is described as follows. Given a graph $(\mathrm{V}, \mathrm{E})$ and a proper function $\mathrm{f}: 2^{\mathrm{V}} \rightarrow\{0,1\}$. The function is said to be proper if (i) $\mathrm{f}(\mathrm{A})=\mathrm{f}(\mathrm{V}-\mathrm{A}) \forall \mathrm{A} \in$ $2^{\mathrm{V}}$, and (ii) if $\mathrm{f}(\mathrm{A})=\mathrm{f}(\mathrm{B})=0$ then $\mathrm{f}(\mathrm{A} \cup \mathrm{B})=0$ for all $\mathrm{A}, \mathrm{B} \in$ $2^{\mathrm{V}}$ s.t. $A \cap B=\varnothing$. The objective is to find a minimum cost forest $H$ in the given graph such that for each $A \in 2^{v}$, if $f(A)$ $=1$ then there is an edge in the forest between $\mathrm{A}$ and $\mathrm{V}-\mathrm{A}$. 
The problem is states as an integer program as follows.

$$
\operatorname{Min} \sum_{e \in \mathrm{E}} c_{\mathrm{e}} \mathrm{x}_{\mathrm{e}}
$$

Subject to

$$
\begin{aligned}
& \mathrm{x}(\delta(\mathrm{S})) \geq \mathrm{f}(\mathrm{S}), \forall \mathrm{S} \text { s.t. } \emptyset \neq \mathrm{S} C \mathrm{~V} \\
& \mathrm{x}_{\mathrm{e}} \in\{0,1\}, \forall \mathrm{e} \in \mathrm{E}
\end{aligned}
$$

where $\delta(\mathrm{S})$ denotes the set of edges having exactly one end point in $\mathrm{S}$ and $\mathrm{x}(\mathrm{F})=\sum_{e \in \mathrm{F}} x_{\mathrm{e}}$.

The forest is given by $\left\{\mathrm{e}: \mathrm{x}_{\mathrm{e}}=1\right\}$.

The Williamson and Goemens algorithm produces a 2 factor approximation for the problem by an ingenious method called primal-dual method. First they consider the LP-relaxation of this problem

$\operatorname{Min} \sum_{e \in \mathrm{E}} c_{\mathrm{e}} \mathrm{x}_{\mathrm{e}}$

Subject to

$$
\begin{aligned}
& \mathrm{x}(\delta(\mathrm{S})) \geq \mathrm{f}(\mathrm{S}), \forall \mathrm{S} \text { s.t. } \emptyset \neq \mathrm{S} C \mathrm{~V} \\
& \mathrm{x}_{\mathrm{e}} \geq 0, \forall \mathrm{e} \in \mathrm{E}
\end{aligned}
$$

and also consider its dual

$$
\operatorname{Max} \sum_{s \subset V} f(S) \cdot y_{\mathrm{S}}
$$

Subject to

$$
\begin{gathered}
\sum_{S: e \in \delta(S)} y_{\mathrm{S}} \leq \mathrm{c}_{\mathrm{e}}: \mathrm{e} \in \mathrm{E} \\
\mathrm{Y}_{\mathrm{S}} \geq 0, \forall \text { S s.t. } . \emptyset \neq \mathrm{S} C \mathrm{~V} .
\end{gathered}
$$

The algorithm computes a greedy solution of the dual problem and in the process it build an approximation of the IP. The greedy algorithm wants to maximize the value of $y_{S}$ for each $S$ such that $f(S)=1$. During the computation a partial solution is a collection of the edges, F, of the graph. Let the connected components of the graph defined by these edges be $\mathrm{C}_{1}, \mathrm{C}_{2}, \ldots \ldots \ldots$ those $\mathrm{C}_{\mathrm{i}}$ are called active if its $\mathrm{f}(\mathrm{)})$-value is 1 . Observe that if any $\mathrm{S} \sqsubset \mathrm{V}$ has $\mathrm{f}(\mathrm{s})=1$, then properness of $\mathrm{f}$ requires that at least one active $C_{i}$ is a subset of $S$. The greedy algorithm considers all edges which connect an active component to another component. One of these edges is selected and added to $\mathrm{F}$ base on the following criterion.

Let edge $\mathrm{e}=(\mathrm{ab})$ connects two components $\mathrm{C}_{1}$ and $\mathrm{C}_{2}$. Then $\mathrm{y}_{\mathrm{c} 1}$ and $\mathrm{y}_{\mathrm{c} 2}$ together can be increased by $\epsilon=\mathrm{c}_{\mathrm{e}}-\sum_{S: e \in \delta(S)} y_{\mathrm{S}}$. Select such edge which yields minimum $\epsilon$ to ensure that the first condition of the dual program remains satisfied for all edges. The final $\mathrm{F}$ is the desired approximation of the IP. Goemans and Willianson show that this gives a 2approximation for some NP-hard problems.

\section{APPLICATION TO THE WIRING PROBLEM AND ITS FAILURE}

In order to solve the wiring problem using primal-dual method we define $f$ as follows. Let $f(S)=1$ iff there are rectangles I and $j$ such that $R_{i} \subset S$ and $R_{j} \subset V-S$, where $R_{i}$, $R_{j}$ denote the vertices on the boundry of the respective rectangles. Following result show that the constrained forest problem subject to this $f$ gives the solution of the wiring problem.

Theorem 1 Let $\mathrm{F}$ be a solution of constrained forest problem subject to $\forall S \subset \mathrm{V}$, if there exists rectangle $\mathrm{R}_{\mathrm{i}}$ and $\mathrm{R}_{\mathrm{j}}$ such that $\mathrm{R}_{\mathrm{i}} \subset \mathrm{S}$ and $\mathrm{R}_{\mathrm{j}} \subset \mathrm{V}-\mathrm{S}$, then $\mathrm{f}(\mathrm{S})=1$, then there exists a connected solution of cost less than or equal to the cost of $\mathrm{F}$.

Proof We consider two cases. If there is a rectangle which touches all the remaining rectangles, then the shortest section of its boundry is the optimal solution. Therefore the nontrivial case the one in which each rectangle is separable from at least one other rectangle (i.e., this rectangle does not share any vertex with another rectangle).In the remainder of this proof we will assume this case.

Suppose $\mathrm{F}$ is not connected. Then there must be a cut through the floorplan, separating its vertices. We will discuss all different kinds of possible partitions to the floorplan one by one. Let a partition divides the whole structure into two sets $\mathrm{R}$ and $\mathrm{S}$. We call an edge e orthogonal to a box $\mathrm{T}$ if and only if $\mathrm{e} \in \delta(\mathrm{T})$, i.e. e has exactly one end point in T. Two boxes are called separable if they don't share any vertex between them.

- When there is one full box in both sets $\mathrm{R}$ and $\mathrm{S}$, the problem definition itself ensures one connection across the partition connecting both components on either sides. This case is shown in figure 2(a)

- When there is at least one full box in one set R but not in S. S has got one connected component of wiring. Please note that each and every box shuld have at least one edge orthogonal to it. Since any box is contained only partially in $\mathrm{S}$, it may have an edge orthogonal to it in R. There should be at least a box partially contained in $\mathrm{S}$ not having orthogonal edge in $\mathrm{R}$ otherwise optimal wiring will lie completely in R. Let $\mathrm{T}$ be such a box. If there is a box separable from $\mathrm{T}$ in $\mathrm{R}$ as shown in figure 2(b), we can redefine partition as $R^{\prime}=R \cup T$ and $S^{\prime}=S$ T. Now an edge will be forced between R' and S'. Let us consider the case when $\mathrm{R}$ contains no box completely which is separable from T. Please note that since $\mathrm{T}$ has no edge orthogonal to it in $\mathrm{R}$, so $\mathrm{T}$ will be connected to any box separable from it completely through S. Please note that in this case any box completely contained in $\mathrm{R}$ shares at least one vertex with $\mathrm{T}$. We do have one component of wiring completely in $\mathrm{S}$ and now we look for edges satisfying requirements for boxes in R. By geometry it should be noted that solution for them can be 
modified without increasing the cost by having all orthogonal edges for these boxes on the boundry of $\mathrm{T}$ as shown in figure 2(c). So now the solution includes component in $\mathrm{S}$ and edges on boundry of $\mathrm{T}$. If both these components are not connected, we have to ensure connectivity between them. A component of wiring not on the boundry of $\mathrm{T}$ will exist in $\mathrm{S}$ if and only if there is a box separable from it in $S$, let us call this box $T_{1}$. Now there should be one box separable from it contained in $\mathrm{R}$ and ofcourse touching $\mathrm{T}$, otherwise it will be special case 2 where a box touches all other boxes. Let $\mathrm{T}_{2}$ be such a box. In this case another partition can be created as shown in figure $2(\mathrm{~d})$ with both components on opposite sides and each set will contain one of $\mathrm{T}_{1}$ and $\mathrm{T}_{2}$ completely. Thus this partition will now ensure connectivity between both sides. Only problem can occur if $\mathrm{T}$ itself is a corner box, i.e. having two edges on the boundry of floorplan. But according to assumption R contains a box completed so T can't be on a corner.

- When no set of the partition contains a box completely, then wiring components on both sets should touch all boxes which isn't possible in case of optimal wiring. Component on one side will be completely redundant in this case. If wiring on $\mathrm{R}$ doesn't touch $\mathrm{T}$, we can define a new partition $\mathrm{S}^{\prime}=$ $\mathrm{S}-\mathrm{T}$ and now one set contains one box completely, and thus connectivity can be ensured across this partition. These cases are shown in figure 2(f).

The primal -dual method solves the problem by picking edges so that the sets having f-value equal to 1 grow into larger sets. This approach fails in the wiring problem because $f$ of every vertex is zero. This gives no starting point to build the solution. This reflects in the fact that $\mathrm{f}$ function defined above is not proper. While $\mathrm{f}\left(\mathrm{R}_{\mathrm{i}}\right)=1, \mathrm{f}(\mathrm{x})=0$ for all $\mathrm{x} \in \mathrm{R}_{\mathrm{i}}$.

\section{VARIATION PRIMAL-DUAL TECHNIQUE AND THEIR FAILURE IN WIRING PROBLEM}

Goemans and Williamson[2] have also discussed approximation algorithms where $\mathrm{f}$ is not required. This was the motivation for looking into this algorithm since we had the difficulty with $\mathrm{f}$ in the first attempt. One of these is for prize-collecting steiner tree problem. In this case each vertex has an associated penalty. The objective is to compute a tree such that its cost plus the sum penalties of all the vertices not on the tree is minimum. Unfortunately this approach does not serve us in solving the wiring problem since there is no appropriate penalty function available. To elaborate on this point consider a vertex $\mathrm{x}$. If a tree covers at least one vertex from each rectangle to which $\mathrm{x}$ belongs, then there is no need to cover $\mathrm{x}$ and no penalty should be charged [5] [6]. On the other hand if all vertices of one such rectangle are missed by a tree, then there should be an infinite penalty.

Blum et.al. [3] have proposed an approximation for minimum steiner tree connecting $k$ vertices. Naveen Garg[4] has improved the approximation factor. These algorithms are also variants of primal-dual algorithm. Once again this approach does not serve well in solving the wiring problems since these do not guarantee which $\mathrm{k}$ vertices will be connected by the algorithm [7] [8] [9] [10]. In our problem we must connect( at least) one vertex from each rectangle.

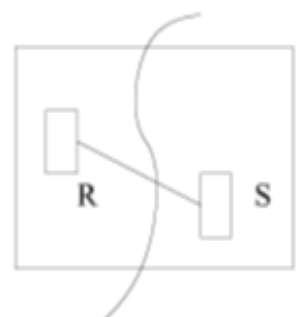

(a)

(c)

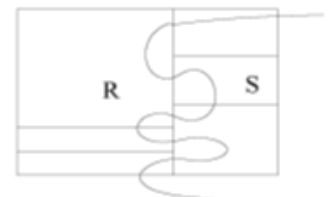

(e)

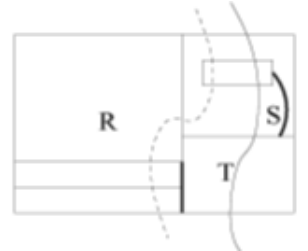

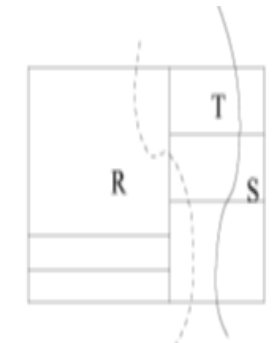

(b)

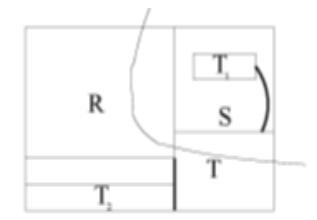

(d)
Figure 2: (a) connection is ensured; (b) partition can be redefined to contain one box completely on either side; (c) wiring for boxes in $R$ can be done on boundary of $T$; (d) connectivity can be enforced between both components; (e) partition can be readjusted to have a box completely on a side if it is not touched by component; (f) wiring will be completely on either side

\section{CONCLUSION}

These approaches have not yielded any satisfactory method to find an approximation solution to the wiring problem. It appears that a very different approach is required to solve this problem. A generalized form of this problem can be stated as follows. Given an edge weighted graph (V, E, W) and a collection of subsets of its vertices $\mathrm{K}$. Find a minimum weight subgraph (V', E') such that $F \cap V^{\prime} \neq \varnothing$ for all $F \in K$. The steiner tree problem is a special case where each set in $\mathrm{K}$ is a singleton.

\section{REFERENCES}

[1] Gabrial Robins and Alexander Zelikovsky. Improved steiner tree approximation in graphs. In SODA 00: Proceedings of the eleventh annual ACM-SIAM symposium on Discrete algorithms, pages 770-779, Philadelphia, PA, USA, 2000. Society for Industrial and Applied Mathematics.

[2] Michel X. Goemans and David P. Williamson. A general approximation technique for constrained forest problems. Siam J. Comput.., 24(2): 296-317, 1995. 
[3] Avrim Blum, R.Ravi, and Santosh Vempala. A constant factor approximation algorithm for the $\mathrm{k}$ mst problem (extende abstract). In STOC 96 : Proceedings of the twenty-eighth annual ACM symposium on Theory of computing, pages 442-448, New York, NY, USA, 1996. ACM Press.

[4] N. Garg. A 3-approximation for the minimum tree spanning $\mathrm{k}$ vertices. In FOCS 96: Proceedings of the $37^{\text {th }}$ annual symposium on Foundation of computer Science,Page 302, Washington, DC, USA, 1996, IEEE computer Society

[5] A. Blum, R. Ravi, and S. Vempala. A constant factor approximation for the k-mst problem. In Proceedings, ACM Symposium on Theory of Computing, 1996.

[6] D. Eppstein. Faster geometric k-point mst approximation. Technical Report 13, University of Califomia, Imine, CA, 1995.
[7] N. Garg and D. Hochbaum. An $\mathrm{O}(\log \mathrm{k})$ approximation algorithm for the $\mathrm{k}$ minimum spanning tree problem in the plane. In Proceedings, ACM Symposium on Theory of Com- puting, 1994.

[8] M. Goemans and D. Williamson. A general approximation technique for constrained forest problems. SIAM J. Comput., 24 296-3 17,1995.

[9] J. Mitchell. Guillotine subdivisions approximate polygonal subdivisions: a simple new method for the geometric kmst problem. In Proceedings, ACM-SIAM Symposium on Dis- crete Algorithms, pages 402408,1996.

[10] R. Ravi, R. Sundaram, M. Marathe, D. Rosenkrantz, and S. Ravi. Spanning trees short and small. In Proceedings, ACM-SIAM Symposium on Discrete Algorithms, 1993. 